\title{
Magnetoelectric coupling of $\mathrm{LaFeO}_{3} / \mathrm{BiFeO}_{3}$ heterostructures
}

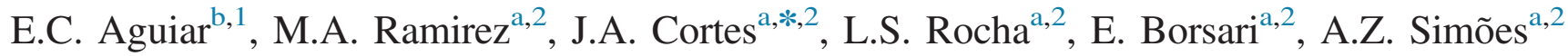 \\ ${ }^{a}$ Universidade Estadual Paulista - UNESP, Faculdade de Engenharia de Guaratinguetá, Av. Dr. Ariberto Pereira da Cunha, 333, Bairro Portal das Colinas, \\ CEP 12516-410 Guaratinguetá, SP, Brazil \\ ${ }^{\mathrm{b}}$ Universidade Estadual Paulista - UNESP, Instituto de Química, Laboratório Interdisciplinar em Cerâmica (LIEC), Rua Professor Francisco Degni s/n, \\ 14800-90 Araraquara, SP, Brazil
}

Received 12 March 2015; received in revised form 24 June 2015; accepted 7 July 2015

Available online 29 July 2015

\begin{abstract}
$\mathrm{BiFeO}_{3}$ (BFO) and $\mathrm{LaFeO}_{3}$ ( $\mathrm{LFO}$ ) heterostructures were obtained at room temperature on $\mathrm{Pt} / \mathrm{TiO}_{2} / \mathrm{SiO}_{2} / \mathrm{Si}(100)$ substrates by chemical solution deposition. The films were coherently grown at a temperature of $500{ }^{\circ} \mathrm{C}$ for $2 \mathrm{~h}$. The magnetoelectric coefficient measurement was performed to show magnetoelectric coupling behavior of such heterostructures. The bottom BFO layer in the heterostructure was able to promote the grain growth of the LFO during the annealing process resulting in huge crystal size. Dielectric permittivity and dielectric loss measurements demonstrated only slight dispersion with frequency due to the lower two-dimensional stress in the plane of the film. Improvement of the $P-E$ hysteresis loop was observed for the heterostructure due the decrease of leakage current caused by the LFO sublayer. Room temperature magnetic coercive field measurements indicate that the LFO and LFO/BFO present different magnetic behavior. The magnetic behavior of the heterostructure is influenced by the crystallite size.
\end{abstract}

(c) 2015 Elsevier Ltd and Techna Group S.r.l. All rights reserved.

Keywords: Ceramics; Thin films; Coatings; Chemical synthesis

\section{Introduction}

Multiferroic materials [1,2] exhibit several ferroic (or antiferroic) orders simultaneously and can be ferroelectric and ferroelastic [3] or ferroelectric and ferrimagnetic [4,5]. The existence of several different parameters in a given material is attractive for memory storage applications as it offers the possibility to store twice as much information in a given memory cell volume, thereby providing an exponential increase in storage density [6]. Ferromagnetic and ferroelectric order parameters are widely used to store binary information in MRAMs [7] and FeRAMs [8], respectively but, unfortunately, ferroelectric ferromagnets (or ferrimagnets) are very scarce and the quest for a material with both large finite polarization and magnetization at

\footnotetext{
*Corresponding author.

E-mail addresses: joalcosu.nal@ gmail.com (J.A. Cortes), alezipo@yahoo.com (A.Z. Simões).

${ }^{1}$ Tel.: +551633019828.

${ }^{2}$ Tel.: + 551231232228 .
}

room temperature is still in progress. To reach this goal, it is neccessary to obtain materials with magnetoelectric coupling. Among all known multiferroics, the only compound that satisfies these criteria is $\mathrm{BiFeO}_{3}$ (BFO). First synthesized in the late 1950s by Kiselev et al. [9,10], BFO was shown to be a G-type antiferromagnet with a Néel temperature of $630 \mathrm{~K}$. Later, Sosnowska et al. showed that the magnetic order of bulk BFO is not strictly collinear and that a cycloidal modulation with a period of $62 \mathrm{~nm}$ is present [11]. Although some methods have been used to decrease the current density leakage of BFO thin films, their electrical behavior is still not ideal [12-15]. Among these promising methods, the bilayered structure consisting of BFO and other ferroelectrics may be a more promising method for decreasing the leakage current density and improving the electrical properties of $\mathrm{BFO}$, because of the bilayered structure. The bilayered thin films consisting of BFO and one other ferroelectric layer have been reported [13-15], such as $\mathrm{Bi}_{1 / 2} \mathrm{Na}_{1 /}$ ${ }_{2} \mathrm{TiO}_{3}, \mathrm{~Pb}(\mathrm{Zr}, \mathrm{Ti}) \mathrm{O}_{3}$. Some interesting results have been demonstrated by such a bilayered structure, such as the reduction in 
current density leakage, the improvement in phase purity, and the enhancement in fatigue behavior, but their polarization value is much lower as compared with the intrinsic polarization of BFO thin films. In the present work, the bilayered thin films consisting of $\mathrm{BiFeO}_{3}$ and $\mathrm{LaFeO}_{3}$ were grown in situ on $\mathrm{Pt} / \mathrm{TiO}_{2} / \mathrm{SiO}_{2} / \mathrm{Si}$ (100) substrates without any buffer layers by the chemical solution deposition method by controlling the thickness of the layers. Lanthanum orthoferrite $\mathrm{LaFeO}_{3}$ (LFO) is a perovskite oxide with semi-conducting behavior [16], and has been extensively studied with reference to several potential applications, such as in gas sensors and as an electrode material in solid-state fuel cells (SOFC) [17]. This oxide crystallizes in an orthorhombically distorted perovskite structure with anti-ferromagnetic characteristics [18]. The particular characteristics of anti-ferromagnetism and an extremely high ordering temperature $\left(T_{\mathrm{N}}\right)$ are very promising for the use of the material in the storage industry, in spin valves, in exchange bias applications and in heterostructures of magnetic/magnetic and magnetic/electric films [19-22]. The magnetoelectric (ME) coefficient $\alpha \mathrm{ME}=d E / d H=d V /(t d H)$ is the most critical indicator for the magnetoelectric coupling properties in multiferroic materials, where $V$ is the induced magnetoelectric voltage, $H$ is the exciting ac magnetic field, and $t$ is the thickness of the sample used for measuring $V$ across the laminate [22]. Among the various approaches to improve the magnetoeletric coupling in $\mathrm{BFO}$ thin films, a combination of antiferromagnetic (AFM) layer consisting of $\mathrm{LaFeO}_{3}$ (LFO) is a promising candidate to improve the coupling between the magnetic and polarization states of the BFO. Antiferromagnetic (AFM) layers play a key role in such magnetoeletronic device applications, where they serve to pin an adjacent ferromagnetic (FM) layer by force of the exchange-bias coupling [23]. The interest in exchange-biased nanostructures has accelerated recently, as a result of advances in fabrication methods and the emergence of novel tools for characterization of magnetic and electrical properties with high spatial resolution. Theoretical models describing exchange-bias recognize the formation of domains in the antiferromagnet as being important to the strength of the exchange coupling. The mechanism responsible for domain formation is not as straightforward as in the case of ferromagnets, since there is no macroscopic demagnetizing field. The fact that a multidomain configuration is usually observed in antiferromagnets is commonly explained by domain stabilization due to lattice imperfections, such as crystalline twins, dislocations, and interstitial atoms. Distortions of the crystalline structure, e.g., due to epitaxial strain, couple to the magnetic order parameter through magnetoelastic effects and are believed to play a key role in the AFM domain formation. The AFM domains are of particular importance to exchange-biased thin film nanostructures comparable in size on the exchange coupling by domain formation in the antiferromagnet. While an extensive body of research exists on magnetic domain formation in FM nanostructures, far less is known about the formation of domains in nanoscale antiferromagnets.

To our knowledge, no reports are available on the magnetoelectric coefficient dependence on the dc bias magnetic field of the LFO-BFO heterostructure. The magnetic and dielectric properties of such thin films crystallized by the polymeric precursor method were investigated for being crucial for device engineering applications. The motivations are as follows: $\mathrm{LaFeO}_{3}$, a room temperature antiferromagnetic, can be used to introduce strain and chemical heterogeneities, and the periodicity of a heterostructure can tailor the collective physical properties of the system.

\section{Experimental procedure}

The thin films were prepared using the polymeric precursor method, as described elsewhere [24]. The films were spin coated (KW-4B, Chemat Technology) on (100) $\mathrm{Pt} / \mathrm{Ti} / \mathrm{SiO}_{2} / \mathrm{Si}$ substrates by a commercial spinner operating at 5000 revolutions $/ \mathrm{min}$ for $30 \mathrm{~s}$. Each annealing layer was pre-fired at $300{ }^{\circ} \mathrm{C}$ for $1 \mathrm{~h}$ in a conventional oven. After the pre-firing, each layer was crystallized in a conventional furnace under static air at $500{ }^{\circ} \mathrm{C}$ for $2 \mathrm{~h}$ up to 10 layers. Phase analysis of the films was performed at room temperature from X-ray diffraction (XRD) patterns recorded on a Rigaku-DMax $2000 \mathrm{PC}$ with $\mathrm{Cu}-\mathrm{K} \alpha$ radiation in the $2 \theta$ range from $20^{\circ}$ to $60^{\circ}$ with $0.3^{\circ} / \mathrm{min}$ steps. For Rietveld analyses, X-ray diffraction data were collected under the following experimental conditions: $40 \mathrm{kV}, 30 \mathrm{~mA}, 20^{\circ} \leq 2 \theta \leq 60^{\circ}, \Delta 2 \theta=0.02^{\circ}, \lambda \mathrm{Cu}$ $\mathrm{k} \alpha$ monocromatized by a graphite crystal, divergence slit $=2$ $\mathrm{mm}$, reception slit $=0.6 \mathrm{~mm}$, step time $=10 \mathrm{~s}$. The Rietveld analysis was performed with the Rietveld refinement program DBWS-941 1. The profile function used was the modified Thompson-Cox-Hasting pseudo-Voigt, in which $\eta$ (the lorentzian fraction of the function) varies with the Gauss and Lorentz components of the full width at half-maximum. The annealed thin film thickness was determined using scanning electron microscopy (SEM) (Topcom SM-300) by checking the crosssection where back-scattered electrons were utilized. Three measurements were taken to obtain an average thickness value. The surface morphology of thin films was measured by atomic force microscopy (AFM) using a tapping mode technique (Nanoscope IIIa-Bruker). Dielectric properties of the capacitor were measured with an HP4192A impedance/gain phase analyzer under zero bias for a film thickness of $270 \mathrm{~nm}$ for the heterostructure, $250 \mathrm{~nm}$ for a BFO layers and $280 \mathrm{~nm}$ for the LFO layer. The crystallite size $(d)$ of the films was calculated using the Scherrer equation $d=k \lambda / \beta \cos \theta$, where $k$ is a constant, $\lambda$ is the wavelength of $\mathrm{X}$ - rays, and $\beta$ is the full width at half-maximum (FWHM) for maximum reflection measured from a slow scan where $\theta$ is the diffraction angle of the main peak.

A $0.5 \mathrm{~mm}$ diameter top Au electrode was sputtered through a shadow mask at room temperature. After deposition of the top electrode, the film was subjected to a post-annealing treatment in a tube furnace under an oxygen atmosphere at $300{ }^{\circ} \mathrm{C}$ for $1 \mathrm{~h}$. Here, the desired effect was to eventually decrease present oxygen vacancies. The $J-V$ measurements were recorded on the Radiant Technology tester in the currentvoltage mode, with a voltage change from 0 to $+10 \mathrm{~V}$, from +10 to $-10 \mathrm{~V}$ and back to $0 \mathrm{~V}$. The hysteresis loop measurements were carried out on the films with a Radiant Technology RT6000HVS at a measured frequency of $60 \mathrm{~Hz}$. 
These loops were traced using the Charge 5.0 program included in the software of the RT6000HVS in a virtual ground mode test device. The magnetoelectric coefficient measurements in the films were attained in a dynamic lockin technique. Additionally, an ac magnetic field up to $10 \mathrm{Oe}$ with frequency of $7 \mathrm{kHz}$ was superimposed onto the dc field. The ac field was produced by a Helmholtz-type coil (180 turns with a diameter of $50 \mathrm{~mm}$ ), driven by an ac current generated by a function generator (Philips PM5192). The amplitude of the ac field was calculated from the driving current measured by a multimeter (Keitley 196 System DMM). Films were located in the magnetic field with the surface perpendicular or parallel to the field direction, for longitudinal and transverse measurements, respectively. The dc magnetic bias field was produced by an electromagnet (Cenco Instruments J type). The time-varying dc field was achieved by a programmable dc power supply (Phillips PM2810 60 V/5 A/60 W). A Hall probe was employed to measure the dc magnetic field. Magnetization measurements were done by using a vibrating-sample magnetometer (VSM) from Quantum Design ${ }^{\mathrm{TM}}$. The magnetoelectric signal was measured by using a lock-in amplifier (EG\&G model 5210) with input resistance and capacitance of $100 \mathrm{M} \Omega$ and $25 \mathrm{pF}$, respectively.

\section{Results and discussion}

Fig. 1 shows the XRD patterns of the heterostructured thin films fabricated in this work as well as for the LFO and BFO film. The XRD spectrum can be separated in two sets of welldefined peaks, one of which belongs to the perovskite BFO and the other to the LFO. The polycrystalline film exhibits a pure perovskite phase and its nature can be attributed to the differences in nucleation energy between the ferroelectromagnetic material and the electrode. Except for the Si (100) and Pt

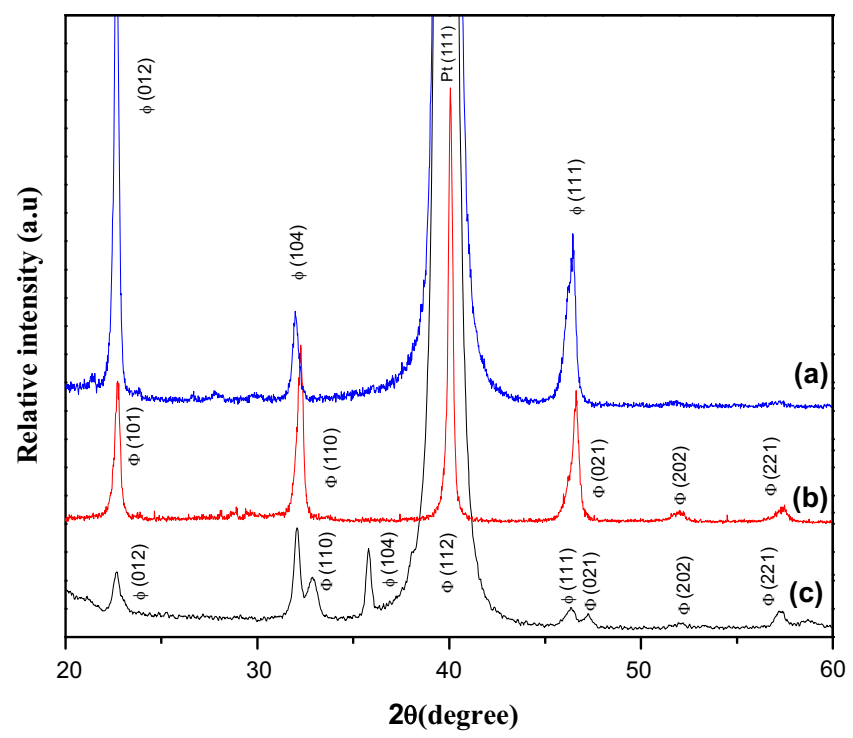

Fig. 1. X-ray diffraction of thin films deposited by the polymeric precursor method and annealed at $500{ }^{\circ} \mathrm{C}$ in static air for $2 \mathrm{~h}$. (a) LFO, (b) BFO and (c) $\mathrm{LFO} / \mathrm{BFO}$.
(111) peaks, no peaks of impure phases such as $\mathrm{Bi}_{2} \mathrm{Fe}_{4} \mathrm{O}_{9}$ and $\mathrm{Bi}_{46} \mathrm{Fe}_{2} \mathrm{O}_{72}$ were found so pure phase $\mathrm{BiFeO}_{3}$ films were obtained by the soft chemical method. The clear identification of these two sets of peak groups without any secondary phase shows that there was little chemical reaction or diffusion between these two constituent layers. The crystal sizes of the phases involved were calculated using the Debye-Scherrer equation, which gave a crystal size of $24 \mathrm{~nm}$ for the BFO film and a crystal size of $37 \mathrm{~nm}$ for the LFO layer. Meanwhile, the crystal size was around $40 \mathrm{~nm}$ for the LFO/BFO layer. These results reveal that the crystal size of the $\mathrm{BFO}$ sublayer in the heterostructure was smaller as compared to that of the sublayer counterpart (BFO). The lattice parameters $(a$ and $c)$ and the unit cell volume $(V)$ of the BFO layers are $a(\AA)=5.6011$, $\mathrm{c}(\AA)=13.6429, V\left(\AA^{3}\right)=371.01$. The atomic positions obtained by Rietveld analyses belong to the ICSD card (PDF 712494) with hexagonal symmetry. In addition, the films are polycrystalline without epitaxy along specific orientation. LFO crystallizes in a distorted antiferromagnet perovskite-structure (orthorhombic, $a=5.557 \AA, b=5.5652 \AA$, and $c=7.8542 \AA$ ) which belongs to the ICSD card (JCPDS file No. 37-1431).

Fig. 2 shows the SEM cross sectional image of the LFOBFO heterostructure. The SEM studies reveal a clear boundary between two different layers, indicating that the LFO sublayer was deposited on the top of the BFO sublayer. Substantial diffusion between two different phases was not observed, which is consistent, with the XRD phase analysis results.

The surface morphologies of the heterostructure and the single layer LFO and BFO are shown in Fig. 3. The heterostructure consists of grains with random size distribution with an average grain size of $68 \mathrm{~nm}$ (Fig. 3c). Fig. 3a shows the single-layered LFO thin films which exhibit a much smaller grain size of $35 \mathrm{~nm}$ while the BFO grain were about $43 \mathrm{~nm}$ in size with high porosity which indicates that the bottom BFO layer in the heterostructure was able to promote the grain growth of LFO during the annealing process. As expected from the larger LFO grain size, the surface roughness of the heterostructure, which was nearly $13 \mathrm{~nm}$, was much

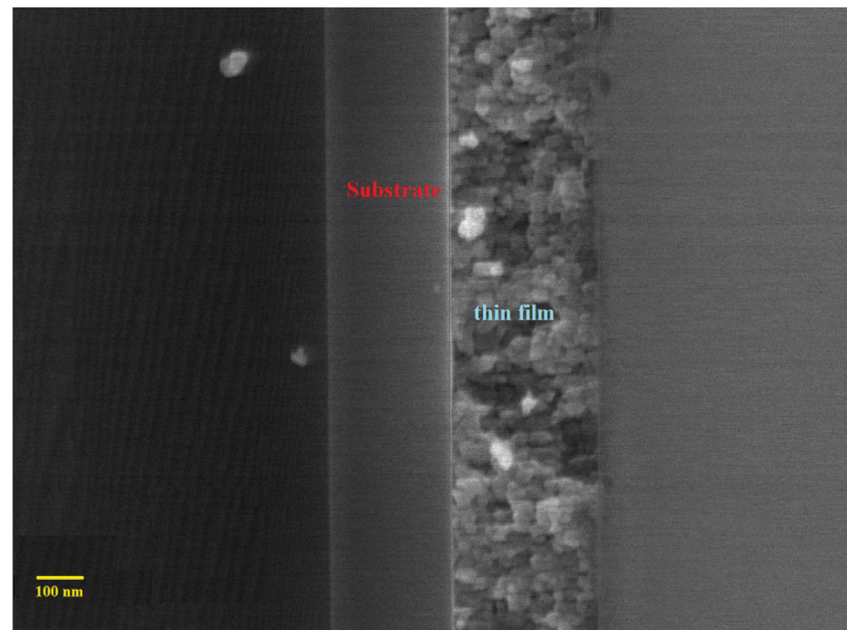

Fig. 2. Cross section of LFO/BFO thin films deposited by the polymeric precursor method and annealed at $500{ }^{\circ} \mathrm{C}$ in static air for $2 \mathrm{~h}$. 
a

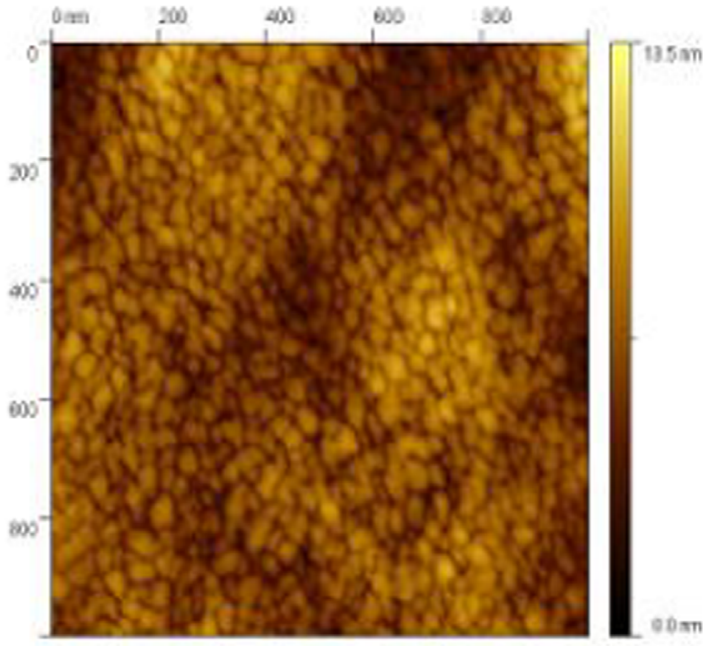

b

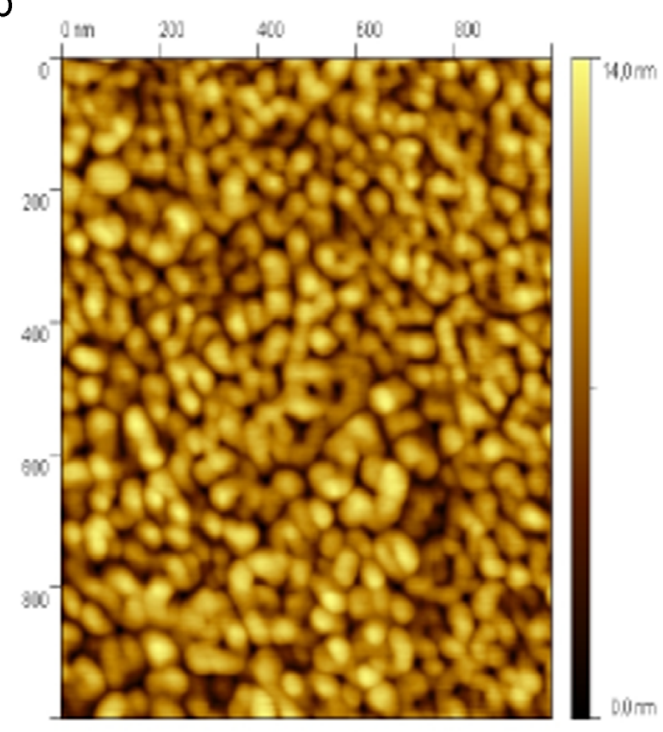

C

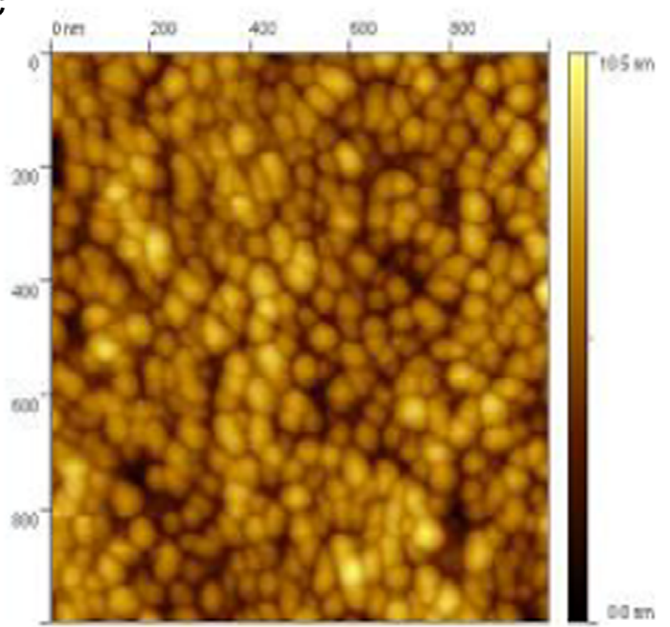

Fig. 3. AFM micrographies of thin films deposited by the polymeric precursor method and annealed at $500{ }^{\circ} \mathrm{C}$ in static air for $2 \mathrm{~h}$. (a) LFO; (b) $\mathrm{BFO}$ and (c) $\mathrm{LFO} / \mathrm{BFO}$ larger than that of single layer LFO $(7 \mathrm{~nm})$ and $(5 \mathrm{~nm})$ of single layer BFO.

Fig. 4a-c shows frequency-dependent dielectric behavior of the $\mathrm{LFO}, \mathrm{BFO}$ and the heterostructured film annealed at $500{ }^{\circ} \mathrm{C}$. The dielectric measurements were carried out at room temperature as a function of frequency in the range of $10 \mathrm{kHz}$
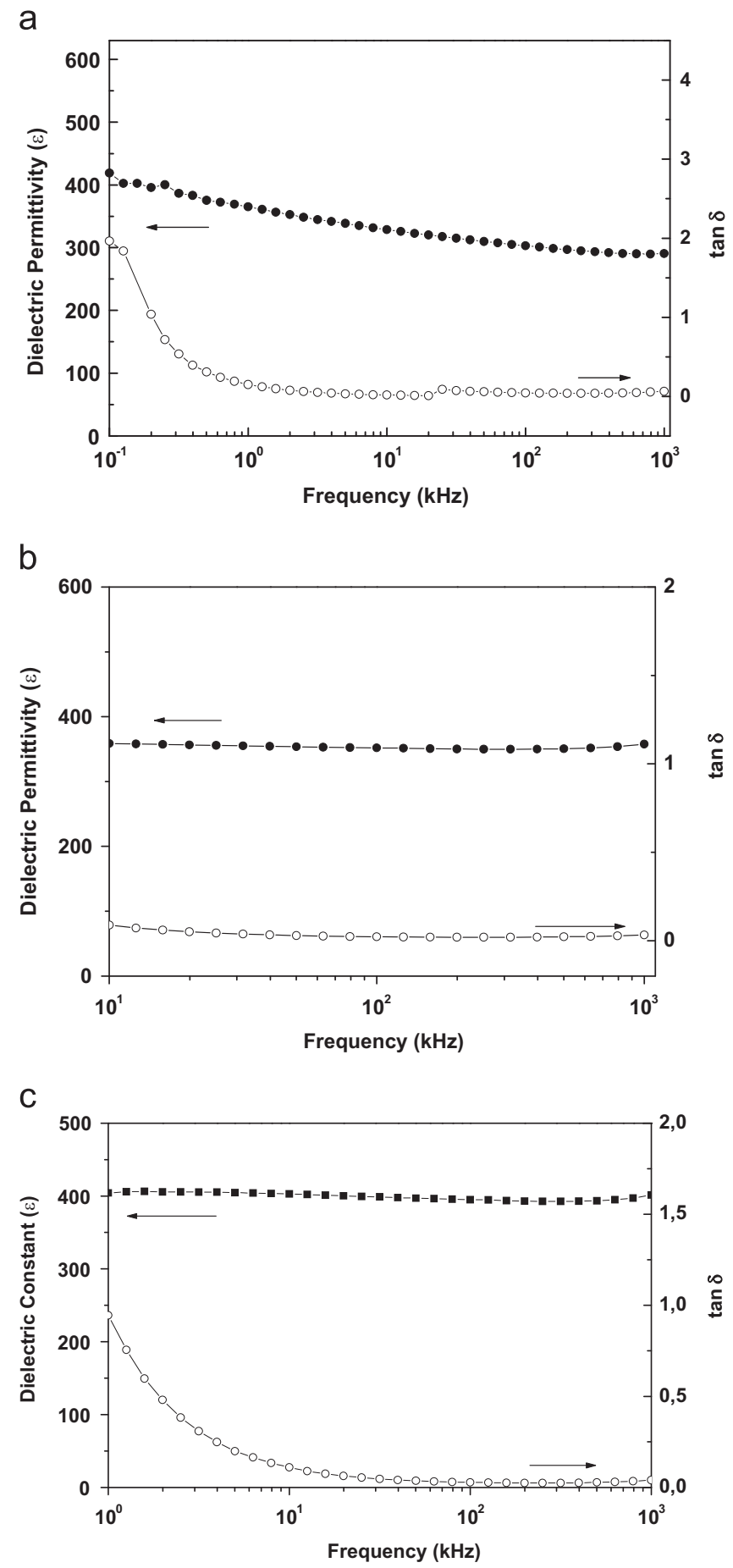

Fig. 4. Dielectric permittivity and dielectric loss spectra of thin films deposited by the polymeric precursor method and annealed at $500{ }^{\circ} \mathrm{C}$ in static air for $2 \mathrm{~h}$. (a) $\mathrm{LFO}$ and (b) $\mathrm{LFO} / \mathrm{BFO}$ as a function of frequency. 
to $1 \mathrm{mHz}$. The films have a small dielectric dispersion at low frequency and the dielectric constants decrease slightly with the frequency, indicating that the films have a good interface with the bottom electrode. The dielectric constant shows very little dispersion with frequency indicating a low concentration of defects at the interface film-substrate. The low dispersion of the dielectric constant and the absence of any relaxation peak in $\tan \delta$ indicate that both, the interfacial polarization of the Maxwell Wagner type and a polarization produced by the electrode barrier can be neglected in the film measurements. The dielectric constant and dissipation factor, at $100 \mathrm{kHz}$, were found to be 290 and 0.06 , respectively for the LFO film (Fig. 4a), 360 and 0.018 for the BFO film. The heterostrutured film had a smaller relative dielectric permittivity (400) when compared with those previously reported in ceramics or films [1,25-31]. The observed improvement in the dielectric permittivity may be associated with higher structural disorder and two-dimensional stress in the plane of the film. The $\tan \delta$ values of the LFO/BFO films changed with increasing frequency suggesting that the films exhibited space charges in low concentration in the sublayers. The tangent loss of the heterostructure is 0.03 and stabilizes after a certain frequency indicating that space charges and defects associated with interfaces have effect in the interface due the improvement in the dielectric permittivity.

Fig. 5 shows the leakage currents density as a function of voltage measured at room temperature. The curve was recorded with a voltage step width of $0.1 \mathrm{~V}$ and elapsed time of $1.0 \mathrm{~s}$ for each voltage. The measured logarithmic current density $(\log J)$ versus the voltage $(V)$ is symmetric and shows two clearly different regions. The insulating properties of the films were found to be dependent on the heterostructure. The leakage current density decreased for the LFO and heterostructured film as compared to pure BFO layer. Such a reduction in leakage current density may be attributed to reduction of the number of electrons injected from the cathode at a rate faster than they can travel through the film. A low threshold electric field was applied in order to overcome the larger repulsion forces that are due to an increase amount of non-neutralized charges in the traps of the LFO film. This study demonstrates that the microstructures of ferroelectric films play an important role in their conductivity properties [32]. Since the conductivity is strongly affected by the characteristics of the film-electrode interface, the lower leakage current observed here may be probably attributed to differences in grain size, density, and less stress in the plane of the film due to differences in the ferroelectric material and the interface. The current density increases linearly with the external voltage in the region of low applied voltage strengths which suggests an ohmic conduction. At higher field strengths, the current density increases exponentially which implies that at least one part of the conductivity results from the Schottky or Poole-Frenkel emission mechanism. The leakage current density at $5.0 \mathrm{~V}$ changes from $10^{-6}$ (LFO-BFO) to $10^{-4} \mathrm{~A} / \mathrm{cm}^{2}$ (BFO) and $10^{-8} \mathrm{~A} / \mathrm{cm}^{2}$ (LFO).

The room temperature $P-E$ hysteresis loops of LFO-BFO and $\mathrm{BFO}$ thin films deposited on Pt electrodes are shown in
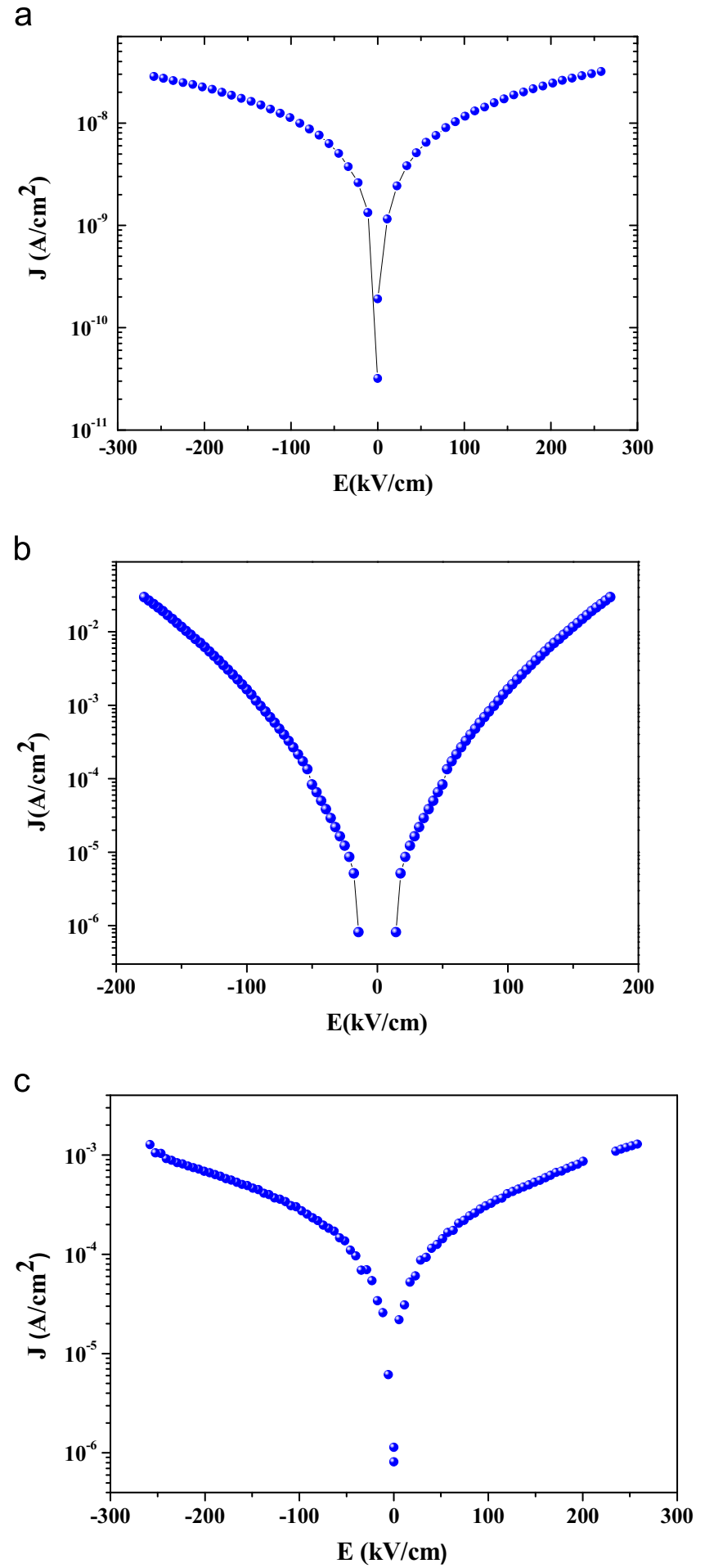

Fig. 5. Leakage current density versus applied voltage of thin films deposited by the polymeric precursor method and annealed at $500{ }^{\circ} \mathrm{C}$ in static air for $2 \mathrm{~h}$. (a) LFO; (b) BFO and (c) LFO/BFO.

Fig. 6a and b. The LFO-BFO film possesses a well saturated and rectangular loop with a $P_{\mathrm{r}}$ of $53 \mu \mathrm{C} / \mathrm{cm}^{2}$ after applying a voltage of $10 \mathrm{~V}$. No sign of leakage has been observed under a measuring frequency of $60 \mathrm{~Hz}$. Possibly, low coercive fields could be expected as the bottom BFO sublayer was subjected for a longer annealing time period resulting in a higher 
a

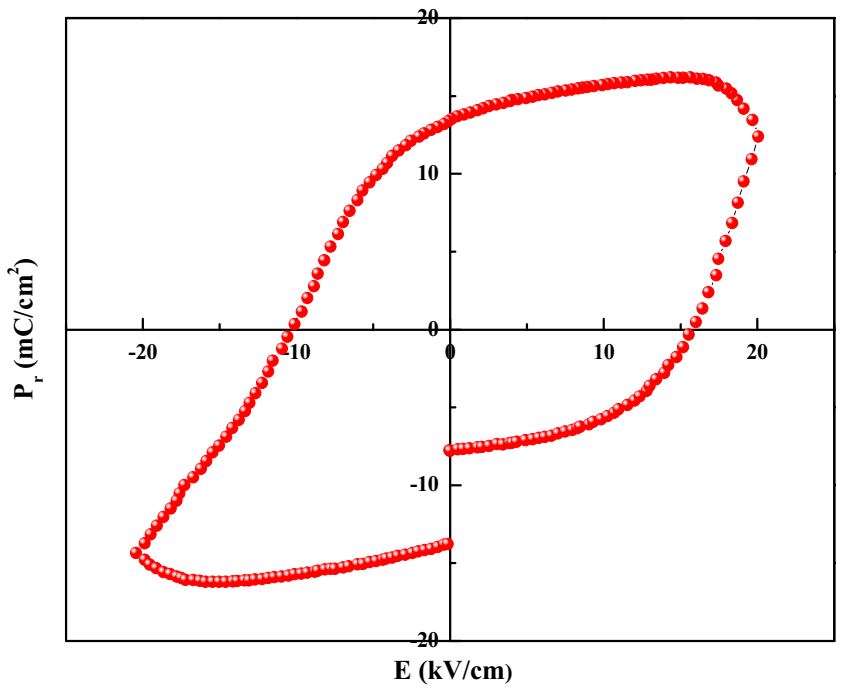

b

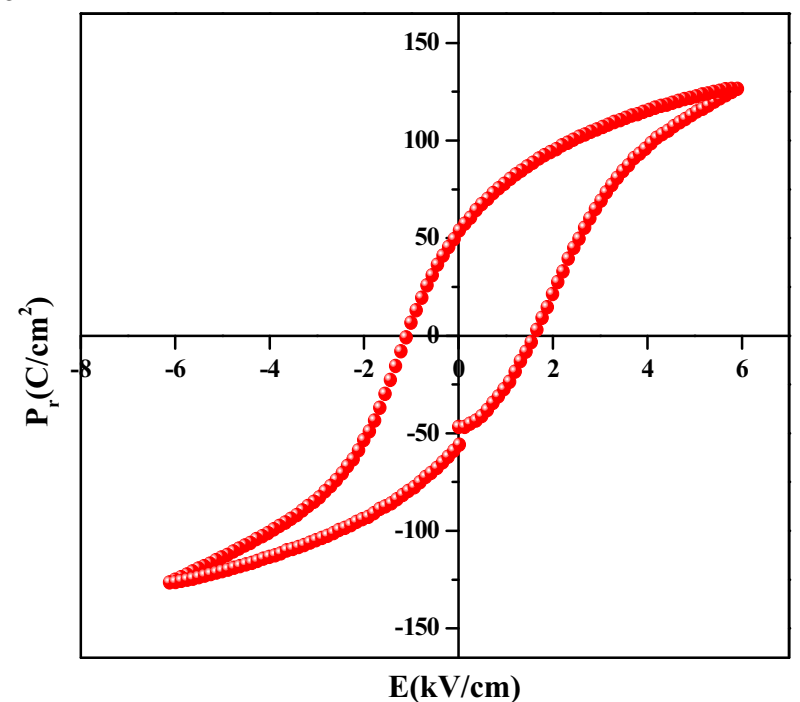

Fig. 6. Hysteresis loop of thin films deposited by the polymeric precursor method and annealed at $500{ }^{\circ} \mathrm{C}$ in static air for $2 \mathrm{~h}$. (a) BFO and (b) LFO/BFO.

concentration of defects and higher leakage. For that reason, new films are being prepared on bottom oxide electrodes to minimize formation of oxygen vacancies between the sublayers. Liu et al. [33] have reported substantially reduced leakage of $\mathrm{BiFeO}_{3}$ films by introducing a $\mathrm{LaNiO}_{3}$ intermediate layer. Although a saturated hysteresis loop was observed, they obtained only a remanent polarization of $26.9 \mu \mathrm{C} / \mathrm{cm}^{2}$ under $1.25 \mathrm{MV} / \mathrm{cm}$. Our results are comparable to those observed in the epitaxial BFO films on a (100) $\mathrm{SrTiO}_{3}$ substrate prepared by the Pulsed Layer Deposition (PLD) method [34]. According to Wang et al., [35] the BFO ferroelectricity originates from the relative displacements of a $\mathrm{Bi}$ ion and a $\mathrm{Fe}-\mathrm{O}$ octahedron along the (111) orientation in epitaxial BFO thin films. The projection polarization along the (110) orientation is larger than the polarization of the (100) orientation. The $P-E$ hysteresis loop of the BFO film shows a $P_{\mathrm{r}}$ of $14 \mu \mathrm{C} / \mathrm{cm}^{2}$ after applying a voltage of $10 \mathrm{~V}$. These high values are not intrinsic, but are induced by high leakage in this structure, as indicated by the roundish shape of the $P-E$ loop. Additionally, space charges can
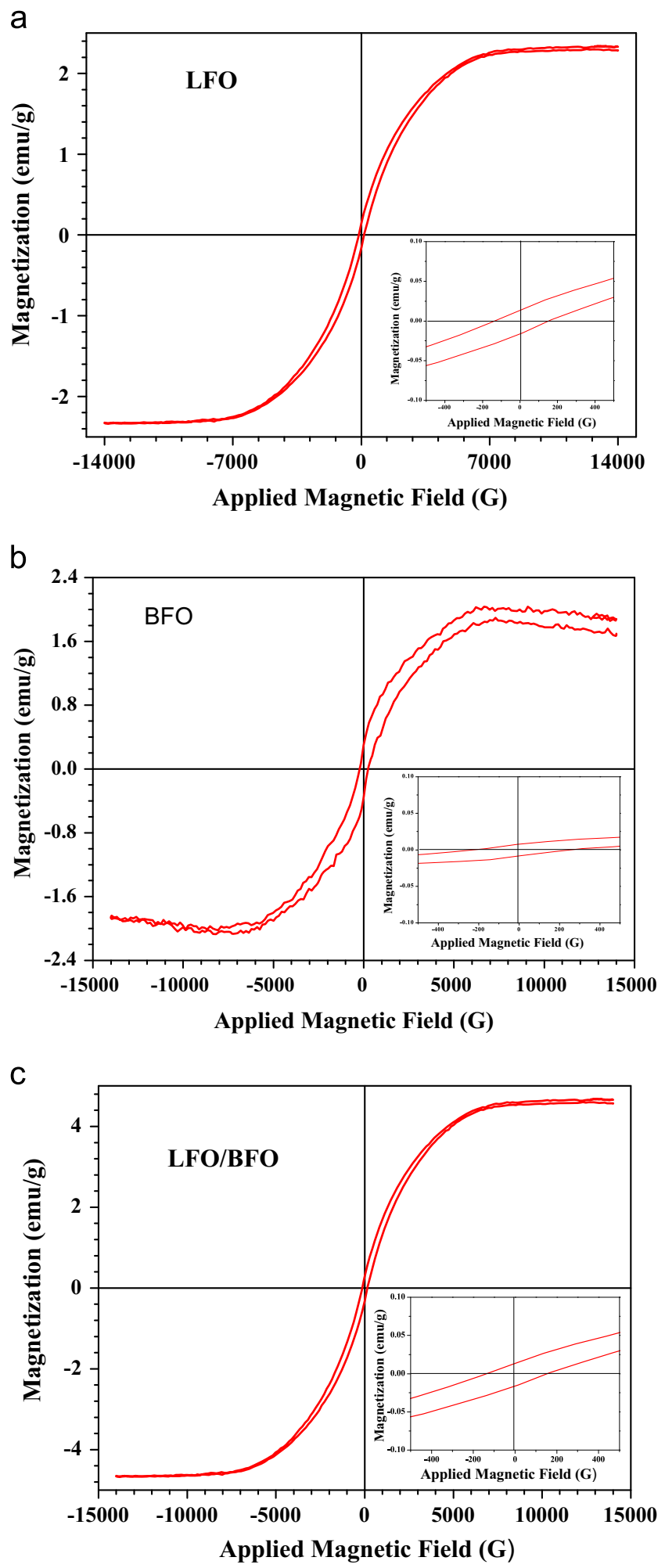

Fig. 7. Field dependencies of the magnetization obtained of thin films deposited by the polymeric precursor method and annealed at $500{ }^{\circ} \mathrm{C}$ in static air for $2 \mathrm{~h}$. (a) LFO; (b) BFO and (c) LFO/BFO. Inset shows the zero field region. 
a

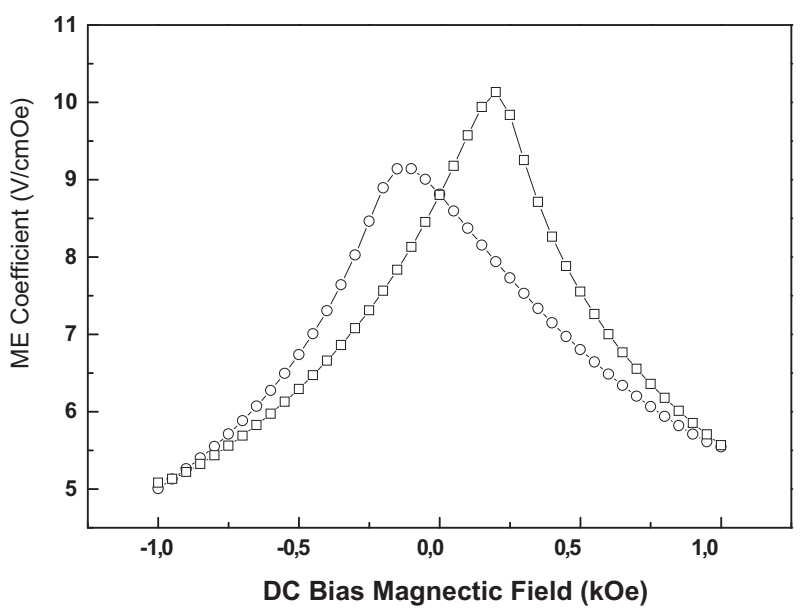

C

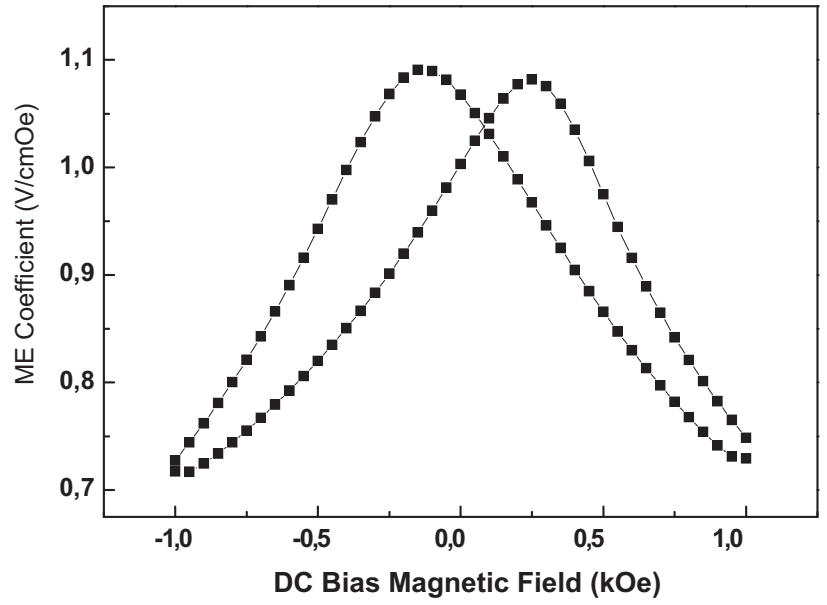

b

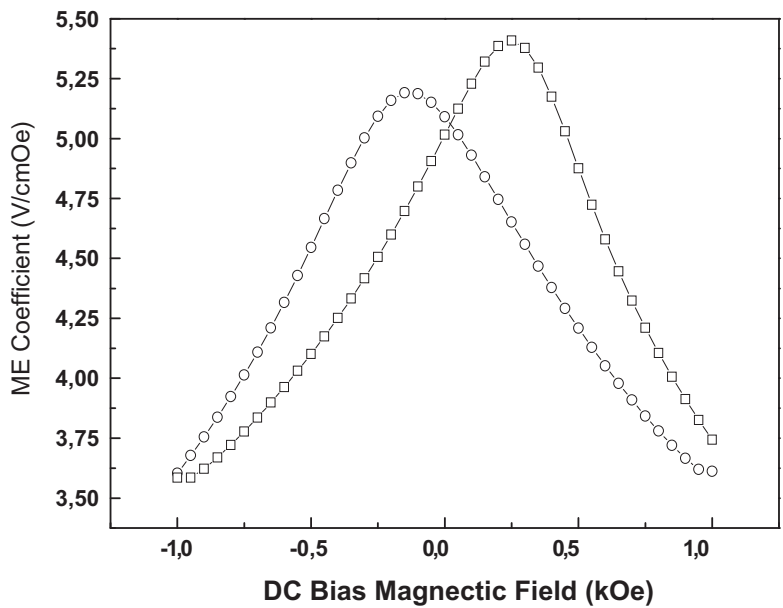

d

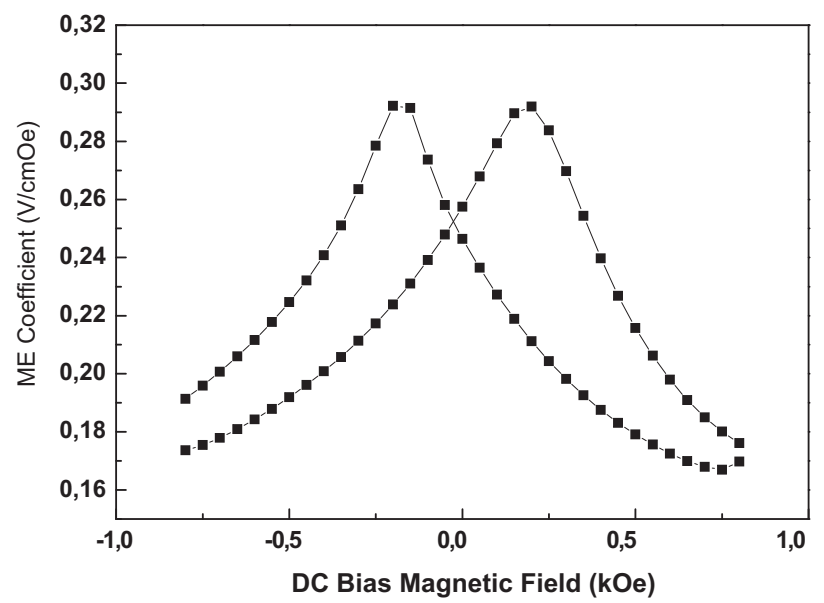

f

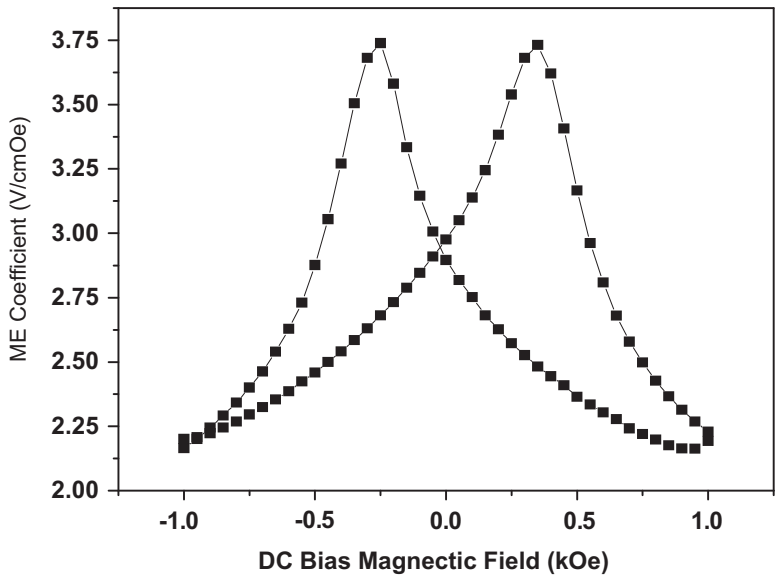

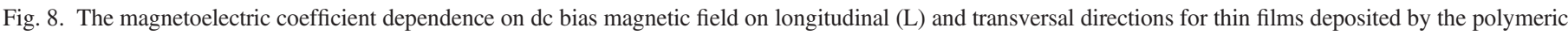

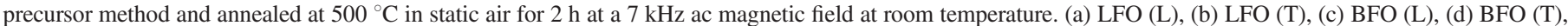
(e) LFO-BFO (L) and (f) LFO-BFO (T).

also contribute to the polarization property. A poor $P-E$ hysteresis loop was observed, as has been typically observed from conductive ferroelectrics and its breakdown with increased bias electric field is due to a large leakage current. Due the absence of hysteresis loop in the LFO film no signal could be measured with the applied voltage and frequency.

Magnetizations $(M)$ versus field $(H)$ loops were recorded at $300 \mathrm{~K}$ (Fig. 7). The magnetic hysteresis loops of the 
single-layered LFO and BFO film and the heterostructure were normalized by the volume fraction of the LFO layer. All films exhibit well-defined hysteresis loops with a saturation magnetization of 2.3, 2.0 and $4.6 \mathrm{emu} / \mathrm{g}$, respectively. The ferromagnetic response suggests that the magnetic behavior is influenced by the crystal size as $M_{\mathrm{S}}$ has been reported to drop according with the reduction in the crystal size [36,37]. The coercive fields $\left(2 H_{\mathrm{c}}\right)$ of the heterostructure and the singlelayered LFO and BFO are 150, 146 and $250 \mathrm{G}$, respectively.

The magnetolectric coefficient versus dc bias magnetic field in the longitudinal and transversal directions reveals hysteretic behavior, as observed in the magnetic field cycles shown in Fig. 8a-f. The maximum magnetoelectric coefficient of $12 \mathrm{~V} / \mathrm{cm}$ Oe in the longitudinal direction is much larger than that previously reported for thin films which was as high as $3 \mathrm{~V} / \mathrm{cm}$ Oe in the same direction at zero fields [38] which is a consequence of the antiferromagnetic axis of LFO/BFO which rotates through the crystal with an incommensurate longwavelength period of $\sim 620 \AA[39,40]$. Early reports showed that the spiral spin structure leads to a cancellation of any macroscopic magnetization and would inhibit the observation of the linear magnetoelectric effect [41]. The hysteretic behavior with peaks at $0.5 \mathrm{kOe}$ comes from the $H_{\mathrm{c}}$ of films being the maximum magnetoelectric coefficient observed when the maximum linear magnetostriction appears. Upon changing the measuring conditions from longitudinal to transverse, $H_{\max }$ changes due to the different demagnetizing field. Significant magnetization $(\sim 0.5 \mu \mathrm{B} / \mathrm{unitcell})$ and a strong magnetoelectric coupling have been observed in epitaxial thin films, suggesting that the spiral spin structure could be suppressed [42]. Also, $\mathrm{BiFeO}_{3}$ and $\mathrm{LaFeO}_{3}$ are G-type antiferromagnets (AFM) with comparable $\mathrm{Fe}$ magnetic moments $(3.75 \mu \mathrm{B}$ and $4.6 \mu \mathrm{B}$ respectively) and high Neel temperatures $(643 \mathrm{~K}$ and $750 \mathrm{~K}$, respectively). Hence, a similar magnetic order well above room temperature was observed. Both materials have a strong tendency to similar crystal structure distortion which in turn is compatible with coupling of lattice modes. Due the high porosity the BFO film present low magnetoelectric coefficient while LFO film is much improved which suggest that the AFM domains serve to pin an adjacent ferromagnetic (FM) layer by force of the exchange-bias coupling in such compound.

\section{Conclusions}

In an attempt to synthesize a composite-type multiferroic structure, a LFO-BFO heterostructure has been created by a chemical solution deposition method. The polycrystalline film consists of both well crystallized BFO and LFO phases with no secondary phases being found. The heterostructure shows a $P_{\mathrm{r}}$ of $53 \mu \mathrm{C} / \mathrm{cm}^{2}$ after applying a voltage of $10 \mathrm{~V}$. Structural defects as high porosity are responsible for the high leakage of the BFO film with low $P-E$ signal. The high dielectric permittivity of the LFO-BFO was mainly due to less structural disorder and less two-dimensional stress in the plane of the film. In-plane magnetization-field curves revealed improvement of magnetization of the heterostructure which was influenced by the crystal size. The maximum magnetoelectric coefficient was nearly $12 \mathrm{~V} / \mathrm{cmOe}$ in the longitudinal direction for the LFO-BFO film. A multiferroic state combining ferroelectricity and weak ferromagnetism is observed. The presented results suggest that such system is a promising candidate to achieve electric switching of the magnetization at room temperature. We hope that this work will motivate further experimental investigations.

\section{Acknowledgments}

The financial support of this research project was from the Brazilian research funding agencies CNPq 573636/2008-7 (National Counsel of Technological and Scientific Development), INCTMN 2008/57872-1 and FAPESP 2013/07296-2 (São Paulo Research Foundation). We would like to thank Professor Elson Longo for facilities.

\section{References}

[1] G.A. Smolenskii, I.E. Chupis, Ferroelectromagnetics, Sov. Phys. Uspekhi 25 (1982) 475.

[2] W. Eerenstein, F.D. Morrison, J. Dho, M.G. Blamire, J.F. Scott, Comment on epitaxial $\mathrm{BiFeO}_{3}$ multiferroic thin film heterostructures, Science 307 (2005) 1203.

[3] A. Vasudevarao, A. Kumar, L. Tian, J.H. Haeni, Y.L. Li, C.J. Eklund, Q. X. Jia, R. Uecker, P. Reiche, K.M. Rabed, L.Q. Chen, D.G. Schlom, Gopalan Venkatraman, Multiferroic domain dynamics in strained strontium titanate, Phys. Rev. Lett. 97 (2006) 257602.

[4] K. Kato, S. lida, Observation of ferroelectric hysteresis loop of $\mathrm{Fe}_{3} \mathrm{O}_{4}$ at 4.2 K, J. Phys. Soc. Jpn. 51 (1982) 1335.

[5] Y. Yamasaki, S. Miyasaka, Y. Kaneko, J.P. He, T. Arima, Y. Tokura, Magnetic reversal of the ferroelectric polarization in a multiferroic spinel oxide, Phys. Rev. Lett. 96 (2006) 207204.

[6] M. Gajek, M. Bibes, S. Fusil, K. Bouzehouane, J. Fontcuberta, A. Barthélémy, A. Fert, Tunnel junctions with multiferroic barriers, Nat. Mater. 6 (2007) 296.

[7] W.J. Gallagher, S.S.P. Parkin, Development of the magnetic tunnel junction MRAM at IBM: from first junctions to a 16-Mb MRAM demonstrator chip, IBM J. Res. Dev. 50 (2006) 5.

[8] M. Dawber, K.M. Rabe, J.F. Scott, Physics of thin-film ferroelectric oxides, Rev. Mod. Phys. 77 (2005) 1083.

[9] P. Royen, K. Swars, Das system Wismutoxyd-Eisenoxyd im Bereich von 0 bis 55 mol\% Eisenoxyd, Angew. Chem. 69 (24) (1957) 779.

[10] S.V. Kiselev, R.P. Ozerov, G.S. Zhdanov, Detection of magnetic order in ferroelectric $\mathrm{BiFeO}_{3}$ by neutron diffraction, Sov. Phys. Dokl. 7 (1963) 742.

[11] I. Sosnowska, T. Peterlin-Neumaier, E. Steichele, Spiral magnetic ordering in bismuth ferrite, J. Phys. C: Solid State Phys. 15 (1982) 4835.

[12] J.G. Wu, G.Q. Kang, H.J. Liu, J. Wang, Ferromagnetic, ferroelectric, and fatigue behavior of (111)-oriented $\mathrm{BiFeO}_{3} /\left(\mathrm{Bi}_{1 / 2} \mathrm{Na}_{1 / 2}\right) \mathrm{TiO}_{3}$ lead-free bilayered thin films, Appl. Phys. Lett. 94 (2009) 172906.

[13] J.G. Wu, G.Q. Kang, J. Wang, Electrical behavior and oxygen vacancies in $\mathrm{BiFeO}_{3} /\left[\left(\mathrm{Bi}_{1 / 2} \mathrm{Na}_{1 / 2}\right)_{0.94} \mathrm{Ba}_{0.06}\right] \mathrm{TiO}_{3}$ thin film, Appl. Phys. Lett. 95 (2009) 192901.

[14] H. Béa, M. Bibes, S. Fusil, K. Bouzehouane, E. Jacquet, K. Rode, P. Bencok, A. Barthélémy, Investigation on the origin of the magnetic moment of $\mathrm{BiFeO}_{3}$ thin films by advanced x-ray characterizations, Phys. Rev. B 74 (2006) 020101(R).

[15] D. Xie, Y. Zang, Y. Luo, X. Han, T. Ren, L. Liu, Structural, ferroelectric, dielectric, and magnetic properties of $\mathrm{BiFeO}_{3} / \mathrm{Bi}_{3.15} \mathrm{Nd}_{0.85} \mathrm{Ti}_{3} \mathrm{O}_{12}$ multilayer films derived by chemical solution deposition, J. Appl. Phys. 105 (2009) 084109. 
[16] E. Traversa, S. Matsushuma, G. Okada, Y. Sadaoka, Y. Sakai, K. Watanabe, $\mathrm{NO}_{2}$ sensitive $\mathrm{LaFeO}_{3}$ thin-films prepared by RF-sputtering, Sens. Actuator B: Chem. 24 (1995) 661.

[17] N.N. Toan, S. Saukko, V. Lantto, Gas sensing with semiconducting perovskite oxide $\mathrm{LaFeO}_{3}$, Physica B 327 (2003) 279.

[18] D. Kuscer, M. Hrovat, J. Holc, S. Bernik, D. Kolar, Some characteristics of $\mathrm{Al}_{2} \mathrm{O}_{3}$ - and $\mathrm{CaO}$-modified $\mathrm{LaFeO}_{3}$-based cathode materials for solid oxide fuel cells, J. Power Sources 61 (1996) 161.

[19] R. Dogra, A.C. Junqueira, R.N. Saxena, A.W. Carbonari, J.M. Filho, M. Moralles, Hyperfine interaction measurements in $\mathrm{LaCrO}_{3}$ and $\mathrm{LaFeO}_{3}$ perovskites using perturbed angular correlation spectroscopy, Phys. Rev. B 63 (2001) 224104.

[20] A. Scholl, J. Stohr, J. Luning, J.W. Seo, J. Fompeyrine, H. Siegwart, J.P. Locquet, F. Nolting, S. Anders, E.E. Fullerton, M.R. Scheinfein, H. A. Padmore, Observation of antiferromagnetic domains in epitaxial thin films, Science 287 (2000) 1014.

[21] J. Nogues, I.K. Schuller, Exchange bias, J. Magn. Magn. Mater. 192 (1999) 229.

[22] D.A. Pan, J. Lu, Y. Bai, W.Y. Chu, L.J. Qiao, Shape demagnetization effect on layered magnetoelectric composites, Chin. Sci. Bull. 3 (2008) 2124.

[23] E. Folven, T. Tybell, A. Scholl, A. Young, S.T. Retterer, Y. Takamura, J.K. Grepstad, Antiferromagnetic domain reconfiguration in embedded $\mathrm{lafeO}_{3}$ thin film nanostructures 10 (2010) 4578Nano Lett. 10 (2010) 4578.

[24] A.Z. Simões, A.H.M. Gonzalez, E.C. Aguiar, C.S. Riccardi, E. Longo, J. A. Varela, Piezoelectric behavior of $\mathrm{SrRuO}_{3}$ buffered lanthanum modified bismuth ferrite thin films grown by chemical method, Appl. Phys. Lett. 93 (2008) 142902.

[25] L.W. Martin, S.P. Crane, Y.H. Chu, M.B. Holcomb, M. Gajek, M. Huijben, C.H. Yang, N. Balke, R. Ramesh, Multiferroics and magnetoelectrics: thin films and nanostructures, J. Phys. C: Condens. Matter 20 (2008) 434220.

[26] G. Catalan, J.F. Scott, Physics and applications of bismuth ferrite, Adv. Mater. 21 (2009) 2463.

[27] K.F. Wang, J.M. Liu, Z.F. Ren, Multiferroicity: the coupling between magnetic and polarization orders, Adv. Phys. 58 (2009) 321.

[28] I. Sosnowska, T. Peterlin-Neumaier, E. Steichele, Spiral magnetic ordering in bismuth ferrite, J. Phys. C: Solid State Phys. 15 (1982) 4835.

[29] C. Ederer, N.A. Spaldin, Weak ferromagnetism and magnetoelectric coupling in bismuth ferrite, Phys. Rev. B 71 (2005) 060401.
[30] X.D. Qi, J. Dho, R. Tomov, M.G. Blamire, J.L. MacManus-Driscoll, Greatly reduced leakage current and conduction mechanism in aliovalention-doped $\mathrm{BiFeO}_{3}$, Appl. Phys. Lett. 86 (2005) 062903.

[31] F.Z. Huang, X.M. Lu, W.W. Lin, X.M. Wu, Y. Kan, J.S. Zhu, Effect of Nd dopant on magnetic and electric properties of $\mathrm{BiFeO} 3$ thin films prepared by metal organic deposition method, Appl. Phys. Lett. 89 (2006) 242914.

[32] X.J. Luo, C.P. Yang, S.S. Chen, X.P. Song, H. Wang, K. Barner, The trap state relaxation related polarization in $\mathrm{CaCu}_{3} \mathrm{Ti}_{4} \mathrm{O}_{12}$, J. Appl. Phys. 108 (2010) 014107.

[33] H. Liu, Z. Liu, K. Yao, Improved electric properties in $\mathrm{BiFeO}_{3}$ films by the doping of Ti, J. Sol-Gel Sci. Technol. 41 (2007) 123.

[34] Z. Cheng, X. Wang, H. Kimura, Z. Ozawa, La and $\mathrm{Nb}$ codoped $\mathrm{BiFeO}_{3}$ multiferoic thin films on $\mathrm{LaNiO}_{3}$ substrates, Appl. Phys. Lett. 9 (2000) 092902-1-092902-3.

[35] Y. Wang, C.W. Nan, Enhanced ferroelectricity in Ti-doped multiferroic $\mathrm{BiFeO}_{3}$ thin films, Appl. Phys. Lett. 89 (2006) 052903.

[36] M.M. Kumar, S. Srinath, G.S. Kumar, Spontaneous magnetic moment in $\mathrm{BiFeO} 3-\mathrm{BaTiO} 3$ solid solutions at low temperatures, J. Magn. Magn. Mater. 188 (1998) 203.

[37] C.S. Liang, J.M. Wu, M.C. Chang, Ferroelectric $\mathrm{BaPbO}_{3} / \mathrm{PbZr}_{0.53} \mathrm{Ti}_{0.47} /$ $\mathrm{BaPbO}_{3}$ heterostructures, Appl. Phys. Lett. 81 (2002) 3624.

[38] J. Li, J. Wang, M. Wuttig, R. Ramess, N. Wang, B. Ruette, A. P. Pyatakov, A.K. Zvezdin, D. Viehland, Dramatically enhanced polarization in (001), (101), and (111) $\mathrm{BiFeO}_{3}$ thin films due to epitiaxialinduced transitions, Appl. Phys. Lett. 84 (2004) 5261.

[39] R. Ramesh, N.A. Spaldin, Multiferroics: progress and prospects in thin films, Nat. Mater. 6 (2007) 21.

[40] I. Sosnowska, T. Peterlin-Neumaier, E. Streichele, Spiral magnetic ordering in bismuth ferrite, J. Phys. C: Solid State Phys. 15 (1982) 4835.

[41] Y.F. Popov, A.K. Zvezdin, G.P. Vorbev, A.M. Kadomtseva, V.A. Murashev, D.N. Racov, Linear magnetoelectric effect and phase transitions in bismuth ferrite $\mathrm{BiFeO}_{3}$, JETP Lett. 57 (1993) 69.

[42] F. Bai, J. Wang, M. Wuttig, J.F. Li, N. Wang, A.P. Pyatakov, A.K. Zvezdin, L.E. Cross, D. Viehland, Destruction of spin cycloid in (111)c-oriented $\mathrm{BiFeO}_{3}$ thin films by epitiaxial constraint: enhanced polarization and release of latent magnetization, Appl. Phys. Lett. 86 (2005) 032511. 